\title{
Manejo perioperatorio del paciente diabético en cirugía podológica
}

\author{
Perioperative management of the diabetic patient in podiatric surgery \\ Andrés José Sánchez Polo ${ }^{1}$, Elisa de Llano Ormazábal ${ }^{2}$ y María del Mar Torrens Darder ${ }^{1}$ \\ ${ }^{1}$ Centro de salud de Santa Ponsa. Atención primaria de Mallorca. Calviá, Mallorca, España. ${ }^{2}$ Actividad privada. Palma de Mallorca, España
}

Palabras clave:

Diabetes, diabetes mellitus, podología cirugía podológica, manejo perioperatorio.

Keywords:

Diabetes, Diabetes Mellitus, podiatry, podiatric surgery, perioperative management.

\section{Resumen}

La diabetes es la endocrinopatía más frecuente, por lo que el podólogo debe estar familiarizado en el manejo prequirúrgico de estos pacientes. Tras historiar y explorar al paciente hay que decidir qué pruebas complementarias se deben prescribir y qué modificaciones en los antidiabéticos se recomiendan en la actualidad. El principal objetivo es mantener unos niveles adecuados de glucemia basal en el preoperatorio, ya que esto disminuye el número de complicaciones intra y posquirúrgicas, mejora la seguridad del paciente y el resultado de la propia cirugía. En cambio, niveles elevados o no controlados de glucemia obligan a diferir la cirugía y derivar al paciente a los servicios de atención primaria o endocrinología. 


\section{INTRODUCCIÓN}

La prevalencia de diabetes (DM) en España es del 13.8 \%, y se estima que hay un $43 \%$ de pacientes diabéticos no diagnosticados ${ }^{1}$. El podólogo es parte fundamental del manejo multidisciplinar del diabético, y entre sus competencias figura la cirugía podológica y el manejo prequirúrgico del paciente diabético, diagnosticado o no, requiere una serie de consideraciones que es fundamental conocer.

La DM es un trastorno metabólico crónico caracterizado por la hiperglucemia, causada por una alteración en la producción de insulina, una resistencia a la insulina o la combinación de ambas $^{2}$. La diabetes tipo 1 (DM1) es el resultado de la destrucción de las células beta pancreáticas, productoras de insulina, por un mecanismo autoinmune, causando déficit en la secreción de insulina; el tratamiento fundamental es la insulinoterapia. Por su parte, la diabetes tipo 2 (DM2), representa el $90 \%$ de los pacientes diabéticos ${ }^{3}$ y es consecuencia de la resistencia periférica a la acción de la insulina que, con el paso de los años, provoca disfunción de las células beta y finalmente déficit en la secreción de insulina; su principal tratamiento farmacológico incluye hipoglucemiantes orales, inyectables no insulínicos e insulina. En la Tabla I se exponen los criterios diagnósticos de la diabetes ${ }^{2}$ y en la Tabla II ${ }^{4}$ se exponen los diferentes tipos de DM.

La diabetes incrementa el riesgo de mortalidad y morbilidad de los pacientes; las principales complicaciones agudas son la hipoglucemia, la cetoacidosis diabética (CAD) y el estado hiperglicémico hiperosmolar (EHH). Las complicaciones crónicas macrovascurales incluyen el infarto de miocardio, el accidente cerebro vascular y la arteriopatía periférica, y las principales complicaciones crónicas microvasculares son la retinopatía, la nefropatía, la coronariopatía y la neuropatía periférica² ${ }^{2}$ Mantener unos niveles óptimos de glucemia basal disminuye el número de complicaciones crónicas y agudas.
Existe una clara asociación entre la hiperglucemia prequirúrgica y los resultados clínicos adversos ${ }^{5}$. Las cirugías producen liberación de hormonas como respuesta al estrés, provocando hiperglucemia intra y postoperatoria ${ }^{6}$, por lo tanto el manejo perioperatorio del paciente diabético debe centrarse en mantener unos niveles óptimos de glucemia para evitar complicaciones como la hiperglucemia extrema, la hipoglucemia y las complicaciones cardiovasculares e infecciosas, principalmente ${ }^{2}$.

Es importante remarcar que se estima que actualmente el $50 \%$ de los diabéticos no están diagnosticados ${ }^{1}$. Estos pacientes presentan hiperglucemia de base y, si a esto se le añade la hiperglucemia por el estrés quirúrgico, el resultado final es que los diabéticos no diagnosticados tienen mayor morbimortalidad”; por tanto, ¿deberíamos realizar glucemia basal de rutina para detectar estos casos?, la respuesta debe englobar cuestiones como la seguridad del paciente, el marco médico legal y el resultado de la propia cirugía, teniendo en cuenta que las pruebas de rutina prequirúrgicas no se recomiendan en la actualidad ${ }^{8}$ (se entiende por exámenes de rutina aquellos solicitados en ausencia de indicación clínica o propósito y está demostrado que no tienen ningún rendimiento significativo, encarecen la atención y generan un dilema de qué hacer frente a un examen alterado cuando este no tiene correlación clínica). El podólogo, con anterioridad a la intervención quirúrgica, debe realizar una evaluación del estado físico del paciente y, según los antecedentes clínicos y la exploración física, determinar las pruebas que se deberían realizar previamente a la intervención ${ }^{9}$.

La cirugía podológica suele ser ambulatoria y electiva en pacientes sin patología de base, o con ella pero sin limitación funcional, y los procedimientos suelen ser de baja o media complejidad, es decir, el marco quirúrgico englobaría pacientes ASA I, ASA II (clasificación de la American Society of Anes-

Tabla I. Criterios diagnósticos de la diabetes ${ }^{2}$.

\begin{tabular}{lccc} 
& Normal & Prediabetes & Diabetes \\
\hline Glucemia basal, en ayunas $(\mathrm{mg} / \mathrm{dl})$ & $70-100$ & $100-125$ & $\geq 126$ \\
\hline Hb1Ac $(\%)$ & $<5.7$ & $5.7-6.4$ & $\geq 6.5$ \\
\hline Glucosa plasmática 2 h tras la carga de glucosa $(\mathrm{mg} / \mathrm{dl})$ & $<140$ & $140-199$ & $>200$ \\
\hline Glucemia al azar + síntomas cardianales* & - & - & $>200$ \\
\hline *Poliuria, polidipsia y polifagia. & &
\end{tabular}

\section{Tabla II. Tipos de diabetes ${ }^{4}$.}

Diabetes tipo 1. Destrucción de células beta. Insuficiencia en la secreción de insulina.

- Inmunomediada

- Idiopática

Diabetes tipo 2. Resistencia periférica a la insulina crónica y progresiva hasta déficit en su secreción

Diabetes gestacional

Maturity Onset Diabetes of the Young (MODY)

Otros tipos de diabetes. Inducidas por fármacos, infecciones virales, inducidas por otras endocrinopatías, etc. 
Tabla III. Grados ASA y ejemplos ${ }^{8-10}$. Grados quirúrgicos según ASECMA-SEDAR-NICE . $^{8}$

Grados ASA

\begin{tabular}{ll}
\hline ASA I. Paciente sano & El paciente ASA I es un paciente sano, que no fuma y no bebe o tiene un mínimo consumo de alcohol \\
\hline $\begin{array}{l}\text { ASA II. Paciente con enfermedad } \\
\text { sistémica leve }\end{array}$ & $\begin{array}{l}\text { Es un paciente con enfermedad sin limitación funcional (como, por ejemplo, paciente fumador, } \\
\text { bebedor social, obeso con índice de masa corporal entre 30 y 40, diabético o hipertenso bien } \\
\text { controlado) o leve enfermedad pulmonar }\end{array}$ \\
\hline $\begin{array}{l}\text { ASA III. Paciente con enfermedad } \\
\text { sistémica grave }\end{array}$ & $\begin{array}{l}\text { Es un paciente con limitación funcional y ejemplos incluyen diabetes y/o hipertensión mal } \\
\text { controladas, enfermedad pulmonar obstructiva, obesidad mórbida, hepatitis activa, dependencia } \\
\text { al alcohol, disminución de la facción de eyección, insuficiencia renal, entre otros }\end{array}$ \\
\hline $\begin{array}{l}\text { ASA IV. Paciente con enfermedad } \\
\text { sistémica grave que constituye una } \\
\text { amenaza constante para la vida }\end{array}$ & $\begin{array}{l}\text { Los ejemplos pueden ser infarto reciente de menos de tres meses, isquemia miocárdica actual, } \\
\text { accidente cerebro vascular, sepsis, entre otros }\end{array}$ \\
\hline $\begin{array}{l}\text { ASA V. Paciente moribundo } \\
\text { Grado 1 (menor) }\end{array}$ & $\begin{array}{l}\text { El ASA V es paciente moribundo cuya supervivencia depende de la cirugía (por ejemplo, } \\
\text { aneurisma aórtico, hemorragia cerebral masiva, entre otros) }\end{array}$ \\
\hline Grado 2 (intermedia) & Grados quirúrgicos \\
\hline Grado 3 (mayor) & Reparación de una hernia, varices, artroscopia de rodilla, amigdelectomía \\
\hline
\end{tabular}

Tabla IV. Ejemplo de evaluación prequirúrgica, modificado ${ }^{6}$.

\begin{tabular}{|c|c|c|c|}
\hline Diabetes conocida & Tipo 1 & Tipo 2 & Otras (MODY, gestacional...) \\
\hline Diabetes no conocida & Sospecha Sí___ & Sospecha No __ & \\
\hline \multirow{3}{*}{ Tratamiento } & \multicolumn{2}{|l|}{ Antidiabéticos orales } & \\
\hline & \multicolumn{2}{|l|}{ Inyectables no insulínicos } & \\
\hline & \multicolumn{2}{|l|}{ Insulina (tipos y dosis) } & \\
\hline \multirow{3}{*}{ Control metabólico } & Glucemia basal ___ & $\mathrm{Hb} 1 \mathrm{Ac}$ & Glucemias habituales ___ \\
\hline & Tendencia a la hipoglucemia & Sí _ـ_ & No __ \\
\hline & \multicolumn{2}{|l|}{$\mathrm{n}^{\circ}$ hipos /Semana ___Mes__ } & \\
\hline \multirow{3}{*}{ Cirugía } & \multicolumn{2}{|l|}{ Diagnóstico } & \\
\hline & \multicolumn{2}{|c|}{ Tipo de intervención y duración } & \\
\hline & \multicolumn{2}{|l|}{ Anestesia } & \\
\hline \multirow{3}{*}{ Órdenes previas } & \multicolumn{2}{|l|}{ Día antes de la cirugía } & \\
\hline & \multicolumn{2}{|l|}{ Día de la intervención } & \\
\hline & \multicolumn{2}{|c|}{ Evaluación por médico familia/endocrino/anestesista } & \\
\hline
\end{tabular}

thesiologist), y cirugías de bajo o medio riesgo según la guía NICE. En la Tabla III se exponen los grados ASA, los grados de riesgo quirúrgico y ejemplos ${ }^{7,8,10}$.

El objetivo del artículo es revisar las recomendaciones sobre la búsqueda de pacientes diabéticos no diagnosticados, cuándo solicitar hemoglobina glicosilada o glucemia basal dentro de las pruebas preoperatorias de estos pacientes, y revisar el manejo farmacológico perioperatorio de la DM2 y entender cuándo es necesario diferir la cirugía.

\section{EVALUACIÓN PREQUIRÚRGICA}

En toda evaluación prequirúrgica se debe realizar una historia clínica completa con el objetivo de determinar si el paciente tiene o no diagnóstico de DM, el tipo, valorar su control metabólico, la presencia o no de complicaciones, el tratamiento que está recibiendo, sus controles glucémicos habituales y saber si ha tenido episodios de hipoglucemia recientemente ${ }^{3-6}$. En la Tabla IV se muestra un ejemplo de este proceso. 
Tabla V. Propuesta para la detección de la diabetes en pacientes no diabéticos ${ }^{3}$.

Edad > 40 años, o 30 años en individuos del sur de Asia

Antecedentes familiares de diabetes

Antecedentes personales de diabetes gestacional

Antecedentes personales de hipertensión arterial

Antecedentes personales dislipemia

Antecedentes personales de prediabetes

Índice de masa corporal $>25$ o $>23$ en individuos del sur de Asia

Pacientes en tratamiento crónico con glucocorticoides

En los pacientes diagnosticados de DM se recomienda realizar ECG como despistaje de infarto previo por la presencia de ondas Q, creatinina sérica para investigar enfermedad renal, electrolitos; sodio y potasio para detectar alteraciones iónicas, glucemia basal y $\mathrm{Hb} 1 \mathrm{Ac}$ con una validez de 3 meses previos a la intervención ${ }^{7-12}$. En caso de $\mathrm{Hb} 1 \mathrm{Ac}$ entre el 6 y $8 \%$ se recomienda no diferir la cirugía; si Hb1Ac es mayor o igual a $8.5 \%$, diferir y derivar a atención primaria o endocrinología para ajuste del tratamiento ${ }^{3-12}$; niveles $\mathrm{Hb} 1 \mathrm{Ac}$ entre el 5 y el $7 \%$ se consideran buen control glucémico; hay que tener en cuenta que la $\mathrm{Hb} 1 \mathrm{Ac}>7 \%$ se asocia con complicaciones infecciosas y la $\mathrm{Hb} 1 \mathrm{Ac}>8 \%$ con complicaciones de la herida quirúrgica ${ }^{5}$.

No se recomienda la búsqueda activa de diabéticos no diagnosticados. Según el programa de actividades preventivas y promoción para la salud (PAPPS) de $2018^{13}$, se propone realizar glucosa basal a partir de los 40 años y cada 4 años como cribado de hiperglucemia, aunque lo más razonable es solicitar glucemia basal o Hb1Ac en pacientes que presenten riesgo de diabetes. En la Tabla $V$ se muestran la propuesta para la detección de la diabetes en pacientes no diabéticos ${ }^{3}$.

Siempre bajo la premisa de individualizar nuestra actuación, se solicitarán más o menos pruebas. Debido a la complejidad y morbilidad que presentan los pacientes diabéticos, se recomienda involucrar en el preoperatorio a los médicos de Atención Primaria u otros especialistas ${ }^{2}$.

En resumen, nuestro paciente tipo sería un paciente DM2, con buen control metabólico; $\mathrm{Hb} 1$ Ac entre 5-7\%, idealmente o $\leq 8.4 \%$ y procedimientos de cirugía menor o mayor ambulatoria y de corta duración.

\section{COMPLICACIONES PERIOPERATORIAS DE LOS PACIENTES CON DIABETES MELLITUS}

Es importante entender las complicaciones que requieren de detección y tratamiento inmediato por presentar una elevada morbimortalidad y, por tanto, no pueden pasar desapercibidas. Partiendo de la base de que a mayor control de la glucemia menores complicaciones cardiovasculares y microvasculares en el paciente diabético, el tratamiento tiene como objetivo mantener unos niveles de $\mathrm{Hb} 1 \mathrm{Ac}$ entre $6 \mathrm{y}$
$6.5 \%$ en pacientes DM y sin comorbilidad asociada, $0<7.5 \%$ si presentan comorbilidad asociada ${ }^{14}$. La contrapartida es que un control intensivo puede provocar hipoglucemias, principalmente en los paciente con DM1 cuyo tratamiento de base es la insulina, y en los DM2 en tratamiento con sulfonilureas, megliptina y/o insulina, todos ellos hipoglucemiantes ${ }^{15}$. En individuos no DM, los niveles de glucemia los regula la insulina pancreática y el glucagón, principalmente; tras la ingesta de alimentos aumentan los niveles de glucosa, el páncreas segrega insulina y la glucosa ingresa en las células normalizando los niveles de glucemia plasmática. En los periodos de ayuno, donde el nivel de glucemia desciende, se inhibe la insulina y aumenta el glucagón provocando síntesis de glucosa hepática. En los pacientes con DM esta homeostasis no existe o es defectuosa, por tanto en situaciones deficitarias de glucosa, como por ejemplo menor ingesta, mayor ejercicio físico, o exceso de insulina inyectada por vía subcutánea, el descenso de glucemia se perpetúa básicamente porque el efecto de los fármacos hipoglucemiantes continúa a pesar del déficit de glucosa y se agudiza más por la inhibición del glucagón. Se considera hipoglucemia niveles de glucemia capilar $\leq 65 \mathrm{mg} / \mathrm{dl}$, pero ante glucemia capilar $\leq 70 \mathrm{mg} / \mathrm{dl} \mathrm{se}$ recomienda iniciar el tratamiento con carbohidratos ${ }^{16}$. Los síntomas suelen ser: temblores, palpitaciones, nerviosismo y malestar; por debajo de $60 \mathrm{mg} / \mathrm{dl}$ aparecen alteración de la consciencia, irritabilidad y ansiedad; por debajo somnolencia, estupor y coma.

No obstante, es importante entender que los síntomas pueden estar enmascarados y no presentarse hasta que el paciente llega al estupor o coma, por lo que ante glucemia capilar de $\leq 70 \mathrm{mg} / \mathrm{dl}$, y debido a las complicaciones potenciales que se pueden derivar de esta situación, se recomienda diferir la cirugía y tratar la hipoglucemia para prevenir que descienda más ${ }^{15}$. En la Figura 1 se muestra en manejo de la hipoglucemia ${ }^{17}$.

Otras de las complicaciones más graves de la diabetes son la CAD y EHH. La CAD se asocia característicamente a DM1 y menores de 65 años y el EHH a DM2 y mayores de 65 años, aunque en situaciones de estrés como infecciones, traumatismos graves o emergencias cardiovasculares, los DM2 pueden desarrollar CAD. Debido al déficit de insulina endógena, total en los DM1 o parcial en los DM2, las células del tejido muscular y periférico ingresan poca glucosa o ninguna; esto provoca la acción de las hormonas contrainsulínicas (glucagón, cortisol y adrenalina principalmente), por lo que se pone en marcha la glucogeogénesis hepática, es decir, se crea un círculo vicioso donde la célula no ingresa glucosa como nutriente, la glucogeogénesis produce glucosa y la glucosa se va acumulando en espacio intravascular, aumentando la glucemia de forma exponencial. Sobrepasado el dintel de $180 \mathrm{mg} / \mathrm{dl}$ de glucemia, el riñón fuerza la glucosuria provocando depleción del volumen; este mecanismo en los DM2 es deficitario por ser un paciente de debut tardío y de mayor edad, por lo que se puede llegar a niveles del glucemia > $1000 \mathrm{mg} / \mathrm{dl}$ en el EHH. En la Tabla VI se exponen los criterios diagnósticos de $\mathrm{CAD}$ y $\mathrm{EHH}^{18}$. El tratamiento se fundamenta en la detección 


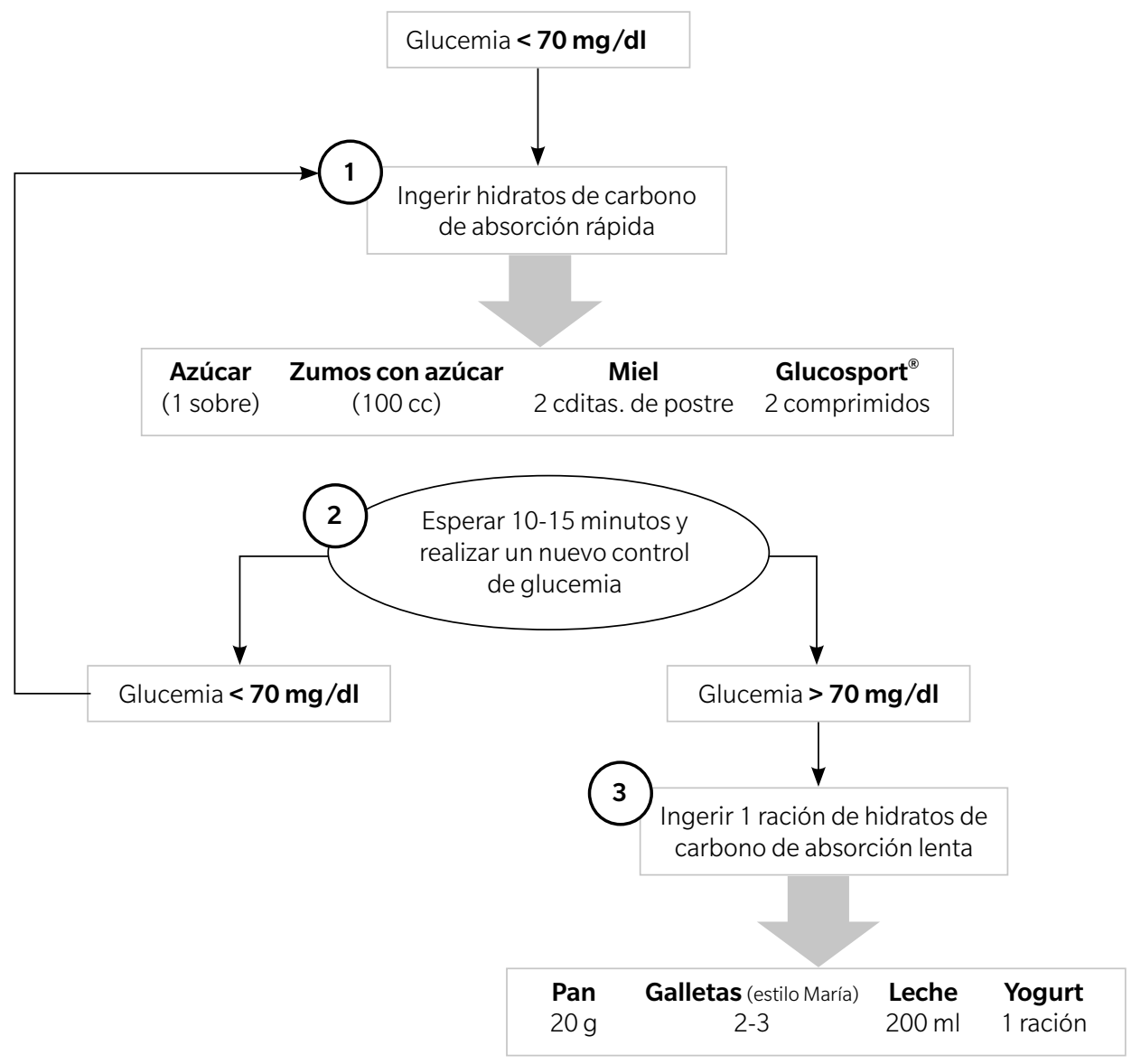

Figura 1. Manejo de la hipoglucemia"17.

Tabla VI. Criterios diagnósticos de la CAD y EHH ${ }^{18}$.

\begin{tabular}{lll} 
& CAD & EHH \\
\hline Glucemia $(\mathrm{mg} / \mathrm{dl})$ & $>250$ & $>600$ \\
\hline Cetonemia $\mathrm{mmol} / \mathrm{l}$ & $>3$ & $<0,6$ \\
\hline $\mathrm{pH}$ & $<7,30$ & $>7,30$ \\
\hline
\end{tabular}

precoz, derivación urgente a un servicio hospitalario y, una vez allí, hidratación intensa, insulinoterapia y restablecimiento de electrolitos.

\section{MANEJO DE LOS NIVELES DE GLUCOSA PREOPERATORIOS}

\section{Objetivos del control glucémico}

Los objetivos generales son evitar la hipoglucemia, la hiperglucemia marcada, prevenir la CAD, el EHHy mantener un adecuado balance de líquidos y de electrolitos. Se ha comprobado que niveles de glucemia prequirúrgico entre 100-180 mg/dl minimizan las complicaciones ${ }^{12} y$, para conseguirlos, es impor- tante dar una serie de recomendaciones por escrito al paciente, tanto prequirúrgicas como posquirúrgicas ${ }^{9}$.

\section{Ayuno}

Se debe intentar modificar lo menos posible la rutina del paciente y minimizar el periodo de ayuno. En los pacientes diabéticos se debe evitar el ayuno prolongado, está comprobado que ayuda a mantener la normoglucemia ${ }^{5-12}$. Para ello, es importante en procedimientos que se requiera anestesia general o anestesia regional con sedación programar la cirugía a primera hora de la mañana y se recomienda ayuno de 8 horas o más para la ingesta de comidas grasas o carne, ayuno de 6 horas para comidas ligeras definidas por tostadas más líquidos claros, y ayuno de 2 horas para líquidos claros, considerados el agua, zumos sin pulpa, te o café. Se ha comprobado que con estas medidas los jugos gástricos son menores y, por tanto, se disminuye el riesgo de broncoaspiración en cirugías que requieran anestesia general, anestesia regional con sedación y analgesia del procedimiento ${ }^{19}$. Parte de las cirugías osteoarticulares que realizamos se encuadran dentro de la anestesia regional, sedación y analgesia, y en este caso el anestesista estará involucrado, junto con el 
podólogo, en el manejo prequirúrgico de estos pacientes. En resumen, se recomienda dieta normal hasta la medianoche y líquidos claros hasta 2 horas previas a la cirugía.

\section{Sobre suspender, o no, los antidiabéticos orales y los inyectables no insulínicos}

La metformina es el primer escalón farmacológico del tratamiento de la DM2, por tanto, una gran mayoría de los pacientes con DM2 estarán en tratamiento con metforfina a no ser que presenten contraindicaciones en su uso. Su mecanismo de acción consiste en disminuir la producción hepática de glucosa y disminuir la resistencia a la insulina en hígado y tejidos periféricos. No aumenta la secreción pancreática de insulina, por tanto el riesgo de hipoglucemias es muy bajo. La metformina puede asociarse a acidosis láctica, es una complicación rara pero puede ser mortal. Está contraindicada en pacientes con filtrado glomerular menor a $30 \mathrm{ml} /$ min y en insuficiencia hepática moderada grave, condiciones que pueden aumentar la concentración plasmática del fármaco y, por tanto, su toxicidad. También es importante tener precaución en la administración de contrastes venosos, ya que pueden disminuir la función renal, y se recomienda no administrar metformina en las 48 horas posteriores a la inyección de contraste endovenoso ${ }^{20}$. Se recomienda la dosis habitual de metformina, tanto el día anterior como en la mañana de la cirugía ${ }^{5,7,21}$.

Las sulfonilureas (glicazida, glimepirida, glipzida) fueron los primeros antidiabéticos orales disponibles para tratar la DM2. Se unen a receptores específicos de las células beta pancreáticas, estimulando la secreción de insulina. Lo hacen tanto en ayunas como con la ingesta de alimentos, provocando liberación basal de insulina. Presentan riesgo de hipoglucemias y su vida media es larga, por tanto el riesgo de hipoglucemia continúa horas después de la toma del medicamento. El riesgo es alto en la insuficiencia renal y hepática, por lo que están contraindicadas en pacientes con filtrado glomerular menor a $30 \mathrm{ml} / \mathrm{min}$ y en la insuficiencia hepática moderada grave $\mathrm{e}^{20}$. Se recomienda la dosis habitual el día anterior a la cirugía, pero suspender la dosis en la mañana de la operación ${ }^{5,7,21}$.

Las glidinas (repaglidina y nateglinida) presentan un mecanismo de acción similar a las sulfonilureas, estimulan la secreción de insulina pero de forma posprandial y su vida media es menor, por lo que le riesgo de hipoglucemia también es menor $^{20}$. Se recomienda la dosis habitual el día anterior de la cirugía y suspender la mañana de la cirugía ${ }^{5,7,21}$.

Glitazonas y pioglitazonas aumentan la sensibilidad a la insulina e inhiben la producción hepática de glucosa, por tanto el riesgo de hipoglucemias es bajo ${ }^{20}$. Se recomienda la dosis habitual tanto el día anterior como en la mañana de la cirugía ${ }^{5,7,21}$.

Respecto a los inhibidores de glucosidasas (acarbosa, miglitol), el mecanismo de acción de estos medicamentos consiste en disminuir e inhibir la absorción de hidratos de carbono a nivel del intestino delgado, no tienen efecto sobre la secreción de insulina y no causan hipoglucemia, aunque hay que tener cuidado ante un paciente que tome acarbosa y presente hipoglucemia, ya que la absorción de hidratos de carbono está disminuida pudiendo perpetuar el estado de hipoglucemia ${ }^{20}$. Se recomienda la dosis habitual tanto el día anterior como en la mañana de la cirugía?.

Los inhibidores de la proteína cotransportadora sodio glucosa tipo 2 (SGLPT-2: canaglifocina, dapaglifozina, empaglifozina) disminuyen la reabsorción de glucosa a nivel de túbulo contorneado proximal, provocando glucosuria y disminuyendo la glucemia. Pueden generar diuresis osmótica, deshidratación e hipotensión ${ }^{7}$. Se recomienda la dosis habitual el día anterior y suspender en la mañana de la cirugía ${ }^{7,21}$. Aunque Duggan y cols. recomiendan suspender los SGLPT-2 el día anterior y el mismo día de la cirugía ${ }^{5}$, partiendo de la base que se debe modificar lo menos posible la rutina del paciente ${ }^{21}$, lo más recomendable es mantenerla dosis habitual el día anterior y suspender en la mañana de la cirugía.

Las incretinas (inhibidores de la DPP-4: alogiptina, linagliptina, saxagliptina, sitagliptina, vidagliptina) presentan un mecanismo de acción que consiste en inhibir la enzima DPP4 encargada degradar al péptido GLP1, que se activa ante la llegada de alimentos al intestino y que estimula a su vez la secreción de insulina, por tanto aumentan la secreción de insulina y disminuyen la secreción de glucagón. Presenta un riesgo de hipoglucemias bajo ${ }^{22}$. Se recomienda la dosis habitual tanto el día anterior como en la mañana de la cirugía ${ }^{5,21}$.

Los inyectables no insulínicos (agonistas GLP-1: albiglutida, dulaglutida, exenatida, liraglitida, lixisenatida) mimetizan la acción de las hormonas denominadas incretinas, responsables entre el 50-70 \% de la secreción de insulina, además de disminuir la secreción de glucagón. Todos se administran por vía subcutánea ${ }^{23}$. Aunque algunos de estos fármacos suelen inyectarse semanalmente, se recomienda la dosis habitual tanto el día anterior como en la mañana de la cirugía ${ }^{7,21}$. En la Tabla VII se resumen las recomendaciones mencionadas con respecto a todos los fármacos descritos.

\section{Insulinas y su manejo prequirúrgico en DM2}

El paciente con DM2 en tratamiento con insulina suele presentar tres opciones de insulinización: a) insulina basal con insulina NPH (Neutral Protamine Hagedorn, insulina de acción intermedia) una o dos dosis, o una dosis de insulina lenta; b) insulina prandial que consiste en la administración de tres dosis de insulina rápida o ultrarrápida antes de las comidas; y c) mezclas de insulinas entre ultrarápida-rápida más insulina intermedia. La primera opción es la de elección actualmente ${ }^{24}$.

Las insulinas ultrarrápidas (lispro, aspart, glulisina) presentan un inicio de acción en menos de 15 minutos, con pico máximo de acción a la hora y duración entre 3 a 5 horas $^{7,24}$. El día antes de la cirugía por la mañana y por la tarde se recomienda mantener las mismas dosis, y en la mañana de la cirugía se recomienda suspender la administración de insulinas ultrarrápidas 5 . 
Tabla VII. Recomendaciones preoperatorias sobre el manejo de fármacos no insulínicos ${ }^{5,7,19}$.

\begin{tabular}{|c|c|c|c|c|}
\hline & Mecanismo de acción & $\begin{array}{l}\text { Riesgo de } \\
\text { hipoglucemia }\end{array}$ & $\begin{array}{l}\text { Dosis el día antes } \\
\text { de la cirugía }\end{array}$ & $\begin{array}{l}\text { Dosis la mañana } \\
\text { de la cirugía }\end{array}$ \\
\hline Metformina & $\begin{array}{l}\downarrow \text { producción hepática de glucosa } \\
\downarrow \text { resistencia a la insulina en hígado y tejidos } \\
\text { periféricos }\end{array}$ & Bajo & Dosis habitual & Dosis habitual \\
\hline Sulfonilureas & $\uparrow$ secreción de insulina & Moderado & Dosis habitual & Suspender \\
\hline Glidinas & $\uparrow$ secreción de insulina & Moderado & Dosis habitual & Suspender \\
\hline Glitazonas & $\begin{array}{l}\downarrow \text { secreción hepática de glucosa } \\
\uparrow \text { sensibilidad a la insulina }\end{array}$ & Bajo & Dosis habitual & Dosis habitual \\
\hline Inhibidores glucosidasas & $\begin{array}{l}\text { Enlentecen la absorción y digestión } \\
\text { intestinal de hidratos de carbono }\end{array}$ & Bajo & Dosis habitual & Dosis habitual \\
\hline SGLPT-2 & $\begin{array}{l}\text { Bloqueo de la reabsorción de glucosa } \\
\text { por el riñón produciendo glucosuria }\end{array}$ & $\begin{array}{l}\text { Bajo, pero riesgo } \\
\text { de cetoacidosis }\end{array}$ & Dosis habitual & Suspender \\
\hline IDPP-4 & $\begin{array}{l}\uparrow \text { secreción insulina } \\
\downarrow \text { producción glucagón }\end{array}$ & Bajo & Dosis habitual & Dosis habitual \\
\hline GLP-1 & $\begin{array}{l}\uparrow \text { secreción insulina } \\
\downarrow \text { producción glucagón }\end{array}$ & Bajo & Dosis habitual & Dosis habitual \\
\hline
\end{tabular}

Tabla VIII. Recomendaciones sobre el manejo prequirúgico de las insulinas en DM25,7,19,21.

\begin{tabular}{llll} 
& $\begin{array}{l}\text { Día antes de la cirugía dosis } \\
\text { de la mañana }\end{array}$ & $\begin{array}{l}\text { Día antes de la cirugía dosis } \\
\text { de la tarde }\end{array}$ & Día de la cirugía dosis de la mañana \\
\hline Insulinas ultrarrápidas & Dosis habitual & Dosis habitual & Suspender \\
\hline Insulina regular & Dosis habitual & Dosis habitual & Administrar el $75 \%$ de la dosis habitual \\
\hline $\begin{array}{l}\text { Insulina intermedia } \\
(\mathrm{NPH})\end{array}$ & $\begin{array}{l}\text { Administrar el } 80 \% \text { de la dosis } \\
\text { habitual }\end{array}$ & $\begin{array}{l}\text { Administrar el } 80 \% \text { de la dosis } \\
\text { habitual }\end{array}$ & $\begin{array}{l}\text { Administrar el } 50 \% \text { de la dosis habitual } \\
\text { si glucemia }>120 \mathrm{mg} / \mathrm{dl} \text {. Si es menor, } \\
\text { suspender }\end{array}$ \\
\hline Insulinas lentas & Dosis habitual & $\begin{array}{l}\text { Administrar el } 80 \% \text { de la dosis } \\
\text { habitual }\end{array}$ & Administrar el $80 \%$ de la dosis habitual \\
\hline
\end{tabular}

La insulina rápida (regular) presenta un inicio de acción en 30 minutos, pico en 2 a 4 horas y duración entre 4 y 8 horas 7,20 . Se recomienda en el día anterior a la cirugía mantener las dosis habituales y en la mañana de la cirugía administrar el $75 \%$ de la dosis habitual ${ }^{20}$.

Las insulinas intermedias (NPH, mezclas de insulinas 30/70 y determir) presentan un inicio de acción a los 60 minutos, con pico de acción entre las 4 y 10 horas y una duración estimada entre 10 y 16 horas $^{7,24}$. Se recomienda en el día anterior de la cirugía tanto mañana y tarde administrar el $80 \%$ de la dosis habitual. En la mañana de la cirugía se recomienda administrar el $50 \%$ de la dosis habitual si la glucemia capilar está $\geq 120 \mathrm{mg} / \mathrm{dl}$ o suspender la administración si es menor ${ }^{5-7}$.

Finalmente, las insulinas lentas (glargina y deglutec) presentan un inicio de acción a las 2 o 4 horas, no presentan pico de acción y tiene una acción durante entre 20 o 24 horas o incluso de 42 horas para degluter ${ }^{24}$. Se recomienda el día anterior a la cirugía por la mañana mantener la dosis y por la noche el $80 \%$ de la dosis habitual. La mañana de la cirugía se recomienda el $80 \%$ de la dosis habitual ${ }^{5-7}$. En la Tabla VIII se resumen las principales recomendaciones sobre el manejo prequirúgico de las insulinas en DM2.

\section{Monitorización de la glucemia}

Como hemos comentado anteriormente, niveles de glucemia prequirúrgicos $\leq 180 \mathrm{mg} / \mathrm{dl}$ disminuyen el número de complicaciones quirúrgicas ${ }^{12}$. Respecto del rango de glucemia adecuado perioperatorio e intraquirúrgico, existe discrepancia. Para Barker y cols. ${ }^{21}$ y Pontes y cols. recomiendan un margen entre 108-180 mg/dl de glucemia capilar; en cambio, Khan y cols. consideran $110-180 \mathrm{mg} / \mathrm{gl}^{12}$, o para Cook y cols. ${ }^{2}$ este rango es de 140-180 mg/dl, aunque todos coinciden en el límite máximo de $180 \mathrm{mg} / \mathrm{dl}$. Se recomienda realizar una glucemia capilar antes de la cirugía, cada 1 o 2 horas durante la misma, y en el posquirúrgico ${ }^{2,6,12}$ para comprobar que se mantiene la glucemia capilar objetivo que, según lo anterior, podemos situarla por encima de $100 \mathrm{mg} / \mathrm{dl}^{6}$ y por debajo de $180 \mathrm{mg} /$ dl. Es importante monitorizar la glucemia capilar, ya que puede cambiar nuestra actuación; ante glucemias $\leq 70 \mathrm{mg} / \mathrm{dl}$ 
Tabla IX. Pautas correctoras para glucemias capilares fuera de objetivo $0^{6,11}$.

\begin{tabular}{ll} 
Glucemia capilar $(\mathrm{mg} / \mathrm{dl})$ & $\begin{array}{l}\text { Análogos de insulina } \\
\text { ultrarrápida (Unidades) }\end{array}$ \\
\hline$<180$ & 0 \\
\hline $180-200$ & 3 \\
\hline $200-250$ & 4 \\
\hline $250-300$ & 8 \\
\hline $300-350$ & 10 \\
\hline$>350$ & 12 \\
\hline
\end{tabular}

hay que tratar la hipoglucemia ${ }^{5,12}$ y diferir la cirugía en nuestro caso, y ante niveles de $\geq 180 \mathrm{mg} / \mathrm{dl}$ se recomienda pauta correctora con insulina rápida subcutánea hasta llegar al objetivo $100-180 \mathrm{mg} / \mathrm{dl}$ o diferir la cirugía y derivar al paciente al centro de salud u hospital, ya que podemos estar ante una hiperglucemia simple, una CAD o EHH que precisarán tratamiento urgente hospitalario. En la Tabla IX se muestran las dosis de pautas correctoras.

En los pacientes DM2 con tratamiento exclusivamente dietético no requieren ninguna terapia especial, aunque habrá que realizar la glucemia capilar antes de la cirugía. Aquellos con glucemias $\geq 180 \mathrm{mg} / \mathrm{dl}$ se tratan con pauta correctora de insulina rápida o diferir la cirugía y derivar al paciente a urgencias ${ }^{6,12}$.

\section{CONCLUSIONES}

La diabetes es la endocrinopatía más frecuente y se estima que el $50 \%$ de los diabéticos no están diagnosticados, por tanto en pacientes no DM pero que presenten riesgo para ello se recomienda solicitar Hb1Ac. En general y en pacientes DM se recomienda realizar ECG, creatinina, sodio, potasio, glucemia basal y Hb1Ac. En caso de $\mathrm{Hb} 1 \mathrm{Ac} \geq 8,5 \%$, se recomienda diferir la cirugía y derivar al paciente para ajuste del tratamiento. Es importante dar instrucciones por escrito tanto prequirúrgicas como posquirúrgicas, respecto a consideraciones generales como a ropa cómoda, calzado adecuado, higiene, etc. Y más concretas sobre el ayuno, y sobre las modificaciones en la posología de los fármacos o de la insulina. Respecto del ayuno se recomienda dieta habitual hasta la noche el día antes de cirugía. Antes de iniciar la cirugía se debe medir la glucemia capilar, repetir la medición cada 102 horas durante la misma y al finalizarla. Ante glucemias $\leq 70 \mathrm{mg} / \mathrm{dl}$ se recomienda diferir la cirugía y tratar de forma enérgica la hipoglucemia. Y ante glucemias $\geq 180 \mathrm{mg} / \mathrm{dl}$ podemos utilizar pauta correctora con insulina rápida, o diferir la cirugía con derivación a servicio de urgencias, siempre a elección del anestesista del equipo. Principalmente en cirugías de bajo riesgo, lo más recomendable es diferir y derivar a urgencias, ya que podemos estar ante una CAD o EHH. El paciente DM es de manejo complejo y suele presentar alta morbilidad, por lo que es conveniente involu- crar en el preoperatorio a otros especialistas, principalmente al anestesista, al médico de familia o al endocrino.

\section{CONFLICTO DE INTERESES}

Los autores declaran que no presentan ningún conflicto de intereses relevante en este artículo.

\section{FUENTES DE FINANCIACIÓN}

No existen fuentes de financiación públicas o privadas en la realización del presente artículo.

\section{BIBLIOGRAFÍA}

1. Soriguer S, Valdés S, Rojo G. El estudio di@betes.es, ¿y ahora qué? Av Diabetol. 2012;28(2):35-3. DOI: 10.1016/j.avdiab.2012.06.001.

2. Cook KD, Borzok J, Sumrein F, Opler JD. Evaluation and perioperative management of the diabetic patient. Clin Podiatr Med Surg. 2019;36(1):83-102. DOI: 10.1016/j.cpm.2018.08.004.

3. Levy N, Dhatariya K. Pre-operativeoptimisation of the surgical patient with diagnosed and undiagnosed diabetes: a practical review. Anaesthesia. 2012;74:58-66. DOI: 10.1111/anae.14510.

4. McCulloch D. Classification of diabetes mellitus and genetics diabetes syndromes. In: Post TW, ed. UpTodate. Waltham, MA: Uptodate, Inc; 2019. Disponible en: https://www.uptodate.com/contents/classification-of-diabetes-mellitus-and-genetic-diabetic-syndromes.

5. Duggan EW, Carlons K, Umpierrez GE. Perioperative hyperglycemia management. Anesthesiology. 2017;126(3):547-60. DOI: 10.1097/ ALN.0000000000001515.

6. Cañada-Sutil V, Maldonado-Areque C, Tapia-Guerreo MJ. Manejo perioperatorio de la diabetes. Protocolo de actuación. Servicios de anestesiología y endocrinología [Internet]. Hospital Regional Universitario de Málaga; 2018 [Acceso en junio de 2020]. Disponible en: http:// www.hospitalregionaldemalaga.es/LinkClick.aspx?fileticket=D_dOotuJrks\%3D\&tabid=673.

7. Pontes JP, Fernadez-Mendez F, Meira-Vasconcelos M, Rodriguez-Batista $\mathrm{N}$. Evaluation and perioperative management of patients with diabetes mellitus. A challenge for the anesthesiologist. Rev Bras Anestesiol. 2018;68(1):75-86. DOI: 10-1016/j.bjane.2017.06.002.

8. Zaballos M. Actualizaciones en pruebas preoperatorias en cirugía ambulatoria. Cir May Amb. 2015;20(1):41-6.

9. Guía práctica de protocolos quirúrgicos en podología [Internet]. Consejo general de colegios oficiales de podólogos; 2009. Disponible en: https://cgcop.es/guias/.

10. Routine preoperative test for elective surgery: NICE (2016) Routine preoperative test for elective surgery. BJU Int. 2018;12(1):12-6. DOI: 10.1111/bju.14079.

11. Kunze S. Evaluación preoperatoria en el siglo xxı. Rev Med Clin Condes. 2017;28(5):661-70.

12. Khad NA, Ghali WA, Cagliero E. Perioperative management of blood glucosa in adults with diabetes mellitus. In: Post TW, ed. UpTodate. Waltham, MA: Uptodate, Inc; 2020. Disponible en: https://www. uptodate.com/contents/perioperative-management-of-blood-glucose-in-adults-with-diabetes-mellitus.

13. Brotons CC, Alemán-Sánchez JJ, Baegas-Banegas JR, Fondón-León C, Lobos-Bejarano JM, Martín-Rioboó E. Recomendaciones preventivas cardiovasculares. Actualizaciones PAPPS 2018. Aten Primaria. 2018;50(1):4-28. DOI: 10.1016/S0212-6567(18)30360-3.

14. Nathan DM. Glycemic control and vascular complications in type 2 diabetes mellitus. In: Post TW, ed. UpTodate. Waltham, MA: Uptodate, Inc; 2020. Disponible en: https://www.uptodate.com/contents/glycemic-control-and-vascular-complications-in-type-2-diabetes-mellitus.

15. Cryer PE. Hypoglycemia in adults with diabtes mellitus. In: Post TW, ed Uptodate. Waltham. MA: Uptodate, Inc; 2020. Disponible en: https:// www.uptodate.com/contents/hypoglycemia-in-adults-with-diabetes-mellitus. 
16. Cryer PE. Physiologic response to hypoglycemia in normal subjects and patients with diabetes mellitus. In: Post TW, ed Uptodate. Waltham. MA: Uptodate, Inc; 2020. Disponible en: https://www.uptodate.com/ contents/physiologic-response-to-hypoglycemia-in-normal-subjects-and-patients-with-diabetes-mellitus.

17. Tratamiento de la hipoglucemia [Internet]. Guía Diabetes Tipo 1. Hospital San Juan de Déu. Barcelona [Acceso en julio de 2020]. Disponible en: https://diabetes.sjdhospitalbarcelona.org/es/diabetes-tipo-1/debut/ tratamiento-hipoglucemia.

18. Hirsch IB, Emmett M. Diabetic ketoacidosis and hyperosmolar hyperglycemis state in adults: Epidemiology and pathogenesis. In: Post TW, ed Uptodate. Waltham. MA:Uptodate, Inc; 2020. Disponible en: https:// www.uptodate.com/contents/diabetic-ketoacidosis-and-hyperosmolar-hyperglycemic-state-in-adults-epidemiology-and-pathogenesis.

19. Barker P, Creasey PE, Dhatariya K, Levy N, Lipp A, Nathanson MH, et al. Association of Anaesthetists of Great Britain and Ireland. Perioperaative managementet of the surgical patient with diabetes 2015. Anaesthesia. 2015;70:1427-40. DOI: 10.1111/anae.13233/full.
20. Apfelbaum JL, Agarkar M, Connis RT, Coté CJ, Nicknovich DG, Warner M. Practique guidelines for preoperative fasting and use of pharmacologic agents to reduce the risk of aspiration: An update report. Anesthesiology. 2011;114(3):495-511. DOI: 10.1097/ALN.0000000000001452.

21. Skully R, Beasley CA, Lutz KW. Current trends in preoperative patient evaluation and management for podiatric surgeons. Clind Podiatr Med Surg. 2003;20(2):213-35. DOI: 10.1016/S0891-8422(03)00004-1.

22. Tran L, Zielinski A, Jende JA, Householder AM, Cole E, Atway S, et al. Fharmacologic treatment of type 2 diabetes: oral medication. Ann Pharmacother. 2015;49(5):540-56. DOI: 10.1177/1060028014558289.

23. Tran L, Zielinski A, Roadch A, Jende JA, Householder AM, Cle $\mathrm{Ce}$, et al. Fharmacologic treatment of type 2 diabetes: injedtable medications. Ann Pharmacother. 2015;49(6):700-14. DOI: 10.1177/106002801573010.

24. Borrás JG, San Martín JE, Mata-Cases M, Gómez-Peralta F, Artola-Menendez A, Fernández-García D, et al. Consenso sobre tratamiento con insulina en la diabetes. Endocrinol Diabetes Nutr. 2018;65(S1):1-8. DOI: 10.1016/j.endinu.2018.01.002. 